\title{
Vacuum Arc Melting Processes for Biomedical Ni-Ti Shape Memory Alloy
}

\author{
De-Chang Tsai ${ }^{1} \mathrm{a}^{*}$ and Chen-Hsueh Chiang ${ }^{2, \mathrm{~b}}$ \\ 11001 Kaonan Highway, Kaohsiung, Taiwan \\ 21001 Kaonan Highway, Kaohsiung, Taiwan
}

\begin{abstract}
This study primarily involved using a vacuum arc remelting (VAR) process to prepare a nitinol shape-memory alloy with distinct ratios of alloy components (nitinol: $54.5 \mathrm{wt} \%$ to $57 \mathrm{wt} \%$ ). An advantage of using the VAR process is the adoption of a water-cooled copper crucible, which effectively prevents crucible pollution and impurity infiltration. Optimising the melting production process enables control of the alloy component and facilitates a uniformly mixed compound during subsequent processing. This study involved purifying nickel and titanium and examining the characteristics of nitinol alloy after alloy melt, including its microstructure, mechanical properties, phase transition temperature, and chemical components.
\end{abstract}

\section{Introduction}

According to the Industrial Technology Research Institute, the estimated value of the 2013 global medical device market was US $\$ 270.3$ billion, with a projected average annual growth rate of $6 \%$. Currently, orthopaedic and dental devices account for $23 \%$ of manufactured medical devices. In addition, shape-memory alloys (SMAs) are required for both orthodontic treatments and minimally invasive surgical procedures. SMAs exhibit a shape-memory effect and hyperelasticity (or pseudoelasticity). Furthermore, they possess characteristics of smart materials and are suitable for biomedical applications, such as orthodontic correction and minimally invasive surgical procedures. SMA is an emerging application material with extremely high added value. The development of production technologies for SMAs and research on relevant technology applications are crucial to establishing Taiwan's biomedical industry, and they are considerably beneficial for enhancing the production value of the domestic metal and precision die industries.

SMAs are smart metal materials that can "remember" their original shape. Olander observed shape-memory effects in 1932. After alloy becomes slightly deformed, the original shape can be recovered by heating the alloy to a certain temperature. [1] When a moderate level of stress is applied to general metal materials, most metals recover their original shape when the external force is withdrawn; however, when the applied stress exceeds the material's load strength, plastic deformation occurs, resulting in permanent deformation. The shape recovery of general metal materials does not exceed $0.1 \%$; by contrast, that of SMAs is approximately 7\%-8\%. Chang and Read (1950) indicated that the characteristics of shape memory are

\footnotetext{
a alvin@mail.mirdc.org.tw

bodalu@mail.mirdc.org.tw
}

determined through phase transformation. As shown in Figure 1, SMAs exhibit hysteresis during the temperature rising and dropping process, [2,3] including austenite start temperature (As), Austenite finish temperature (Af), martensite start temperature (Ms), and martensite finish temperature (Mf). The shape-memory effect refers to the phenomenon of a metal material recovering its original shape at high temperatures; when heated to temperatures above Af, the metallic phase structure transforms from martensite to austenite, demonstrating shape memory. However, when the temperature falls below Mf, the metallic structure is transformed from austenite to martensite, thereby recovering its low-temperature shape. The shape-memory effect of recovering high- and low-temperature shapes varies according to the material components and production process of the alloy.

Another characteristic of SMAs is pseudoelasticity. [2,3] When a SMA is affected by stress, diffusionless phase transition occurs in the material. When the external force is withdrawn, the atoms return to their original positions, recovering the metal to its original shape (Figure 2(c)). Generally, at temperatures higher than Af, shape-recovery occurs when the temperature is lower than the stress-induced martensite finish temperature (Md). As shown in Figure 2(a), when the temperature is lower than Af, residual strain is still present after the stress is withdrawn. If the temperature is below Ms, the residual strain becomes great, as shown in the curves in Figure 2(b). The metal must be heated to a temperature greater than Af to be free of strain through the aforementioned shape-memory effect. [4]

Nitinol is biocompatible. Ryhanence et al. conducted an in vitro experiment and showed that nitinol is nontoxic [5] and does not inhibit cell proliferation. In an in vitro experiment, Wever et al. showed that nitinol is nontoxic 
and does not cause allergic reactions or exert influence on the genes. Moreover, the properties of nitinol are similar to those of 316 stainless steel. [6] Shabalovskaya [7] observed that, according to over 10 years of in vivo research on nitinol, nitinol does not cause any allergic reaction. Furthermore, Shabalovskaya found no corrosion and invasion around explants, and no traces of alloy in surrounding tissues. The bioaffinity performance of nitinol is primarily determined by a passive film composed of $\mathrm{TiO} 2$ on the alloy surface. As long as the passive film on the alloy is intact, corrosion can be prevented because the $\mathrm{TiO} 2$ oxide film is very stable [8]. Although some ion release may occur in the passive state, the released concentration is not sufficiently high to cause toxicity, allergic reaction, or influence on genes.

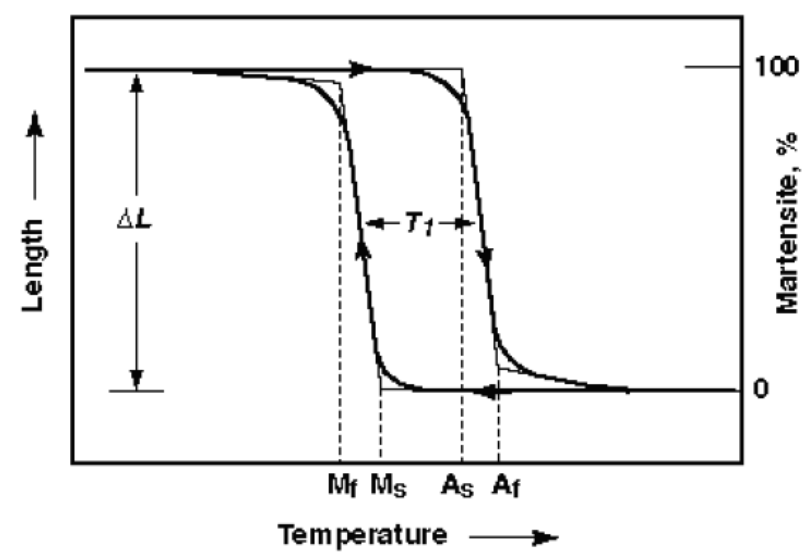

Fig.1 Sketch of phase transition temperature of shape memory alloy

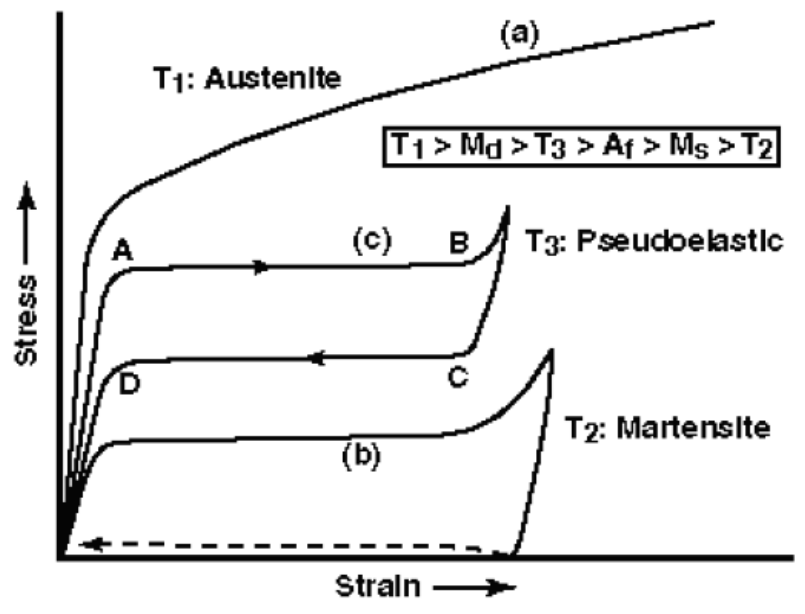

Fig.2 Sketch of stress-strain of shape memory alloy

\section{Experimental method}

The experiments were conducted in 5 steps: (1) production of nitinol specimens; (2) microstructure analysis; (3) mechanical property test; (4) phase change temperature measurement; and (5) biocompatibility test. To produce the nitinol specimens, vacuum arc melting was used to purify the nickel and titanium. The purified metals were subsequently subjected to alloy melting. Figure 3 shows the required experimental equipment. In the microstructure analysis, the alloy composition was detected using a glow discharge spectrometer. A metallographic microscope and a scanning electron microscope were used to observe the microstructures. X-ray diffraction (XRD) was performed to analyse the effect of the alloy components and production process conditions on the phase composition. For the mechanical property test, a universal testing machine was used to identify the effect of the nitinol melt and processing mechanisms on the alloy's mechanical properties according to the ASTM F2516 Standard Test Method for Tension Testing of Nickel-Titanium Superelastic Materials. To measure the phase change temperature, differential scanning calorimetry (DSC) was employed to analyse the phase change temperature of nitinol during the temperature rising and dropping process and to understand the effect and relationship of production process parameters according to the ASTM F2004 Standard Test Method for Transformation Temperature of Nickel-Titanium Alloys by Thermal Analysis. Finally, for the biocompatibility test, cell cultivation, classification, and proliferation were conducted based on the standard ISO 10993 Biological Evaluation of Medical Devices and described quantitatively based on methods for evaluating clinical usability.

\section{Results and Discussion}

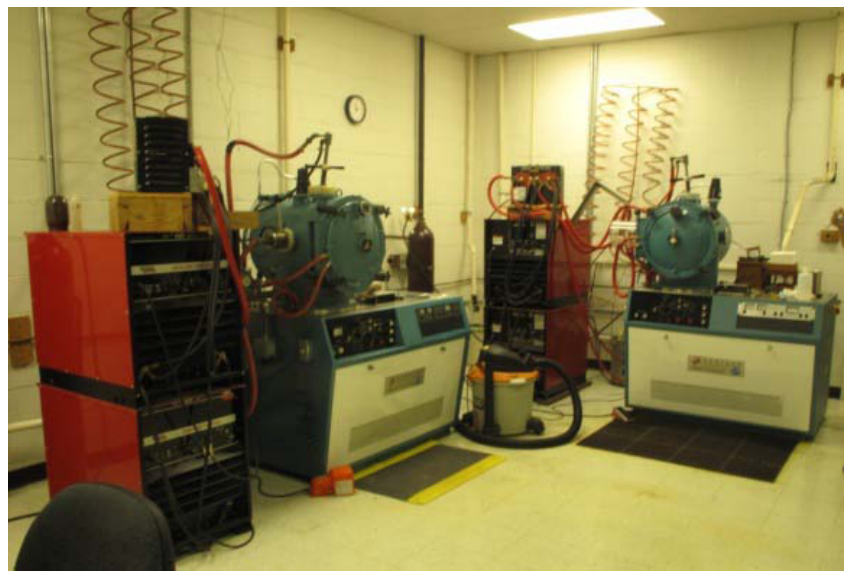

Fig.3 The equipment of vacuum arc remelting

This discussion is presented in 2 sections: (1) vacuum arc remelting (VAR) process, which explores the purification and alloy melting during the nitinol production process; and (2) exploration of the chemical components, microstructure, mechanical properties, and phase change temperatures of nitinol after alloy melt. 


\section{Nitinol VAR process}

The melting point of nitinol is approximately $1580 \mathrm{~K}$. In a molten state, titanium is highly active and oxidation occurs easily. Thus, nitinol is generally melted in high-vacuum inert-gas condition. Table 1 shows the parameter settings used in this study. The vacuum was controlled at $2.0 \times 10-5$ torr and the arc current was set at 100-250 A. Ingot remelting was performed using nonconsumable electrodes, to remove impurities and reduce the gas content $(\max . \mathrm{O}: 0.05 \mathrm{wt} \%$; N: $0.05 \mathrm{wt} \%$; $\mathrm{C}$ : $0.05 \mathrm{wt} \%$; and $\mathrm{H}: 0.005 \mathrm{wt} \%)$. Finally, an electric arc furnace was used to remelt the alloy 3 to 6 times to achieve the required component specifications (homogeneous composition and ASTM F2063 standard) of the nitinol melt. Figure 4 shows the purified and melt nickel, titanium, and nitinol. Table 2 shows the results of the inductively coupled plasma (ICP) analysis of the nitinol. The results conform to the regulations of ASTM F2063.

Table 1 The related experimental parameters of vacuum arc remelting process

\begin{tabular}{cccc}
\hline Crucible & $\begin{array}{c}\text { Cooling } \\
\text { rate } \\
(\mathrm{L} / \mathrm{min})\end{array}$ & $\begin{array}{c}\text { Vacuum } \\
\text { (torr })\end{array}$ & $\begin{array}{c}\text { Current } \\
\text { (A) }\end{array}$ \\
\hline Copper & 30 & $2.0^{*} 10^{-5}$ & 250 \\
\hline
\end{tabular}

Table 2 The ICP analysis of nitinol alloy

\begin{tabular}{c|ccccc}
\hline Alloy & \multicolumn{5}{|c}{ Composition (wt\%) } \\
\hline \multirow{2}{*}{ Nitinol } & $\mathrm{N}$ & $\mathrm{O}$ & $\mathrm{C}$ & $\mathrm{S}$ & $\mathrm{H}$ \\
\cline { 2 - 6 } & 0.001 & 0.035 & 0.013 & 0.001 & 0.0009 \\
\cline { 2 - 6 } & $\mathrm{Co}$ & $\mathrm{Cu}$ & $\mathrm{Cr}$ & $\mathrm{Fe}$ & $\mathrm{Nb}$ \\
\cline { 2 - 6 } & $\mathrm{N} . \mathrm{D}$. & 0.0062 & 0.0086 & 0.0226 & $\mathrm{~N} . \mathrm{D}$. \\
\hline
\end{tabular}

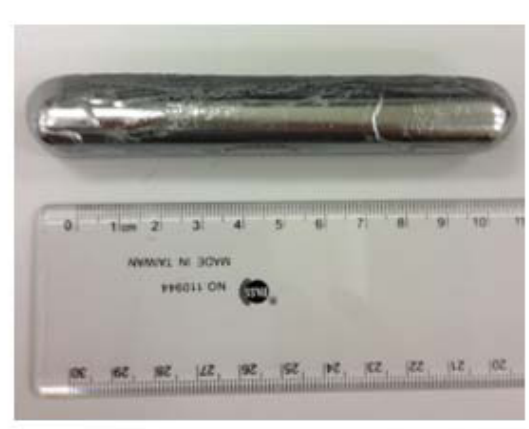

(a)

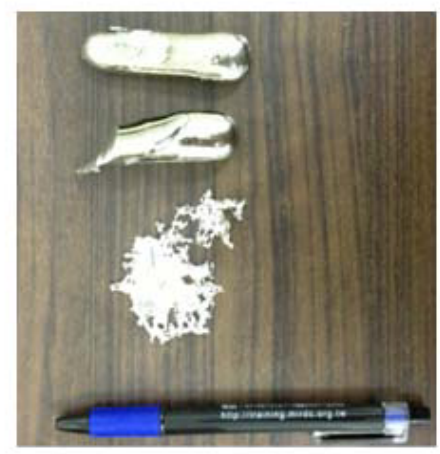

(b)

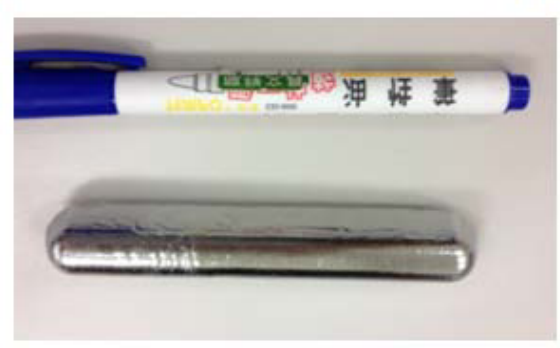

(c)

Fig.4 Sketches of remelted specimen(a) pure nickel (b) pure titanium (c) nitinol alloy 


\section{Physical and chemical property analysis of nitinol}

Figure 5 is metallographic microscope photograph of the horizontal and vertical sections of the nitinol. From the metallographic photograph, we can find the grain structure is mainly column grain structure and the grain size is thinner. The OM photograph shows the phase of nitinol is austenite. The XRD analysis results indicate that after melting, the nitinol consisted primarily of the austenite phase with a B2 structure and featured intermetallic of TiNi3 (Figure 6). According to the phase change temperature measurements, the Ms of nitinol (Ni: 55.9 wt\%) was approximately $-19.7{ }^{\circ} \mathrm{C}$ and the As was approximately $-12.7{ }^{\circ} \mathrm{C}$ (Figure 7 ). These results match those observed in the austenite phase of nitinol at room temperature.

Figure 8 shows the mechanical property analysis results of the nitinol at room temperature $\left(25^{\circ} \mathrm{C}\right)$ and at human-body temperature $\left(37^{\circ} \mathrm{C}\right)$. The elongation and tensile strength test results show that increasing the temperature of the nitinol ingot caused an increase in elongation (from $14.5 \%$ to $15.6 \%$ ) and tensile strength (from 545 to $565 \mathrm{MPa}$ ). Figure 8(b) shows the hyperelastic recovery effect of the nitinol ingot. When the tensile stress reached $6 \%$ strain, the stress was released with a residual strain of $0.36 \%$ and $0.38 \%$. Figures 9 and 10 show the biocompatibility test results. The polished surface of the nitinol was used as an experimental sample, and a tissue culture plate group was used as a control sample. As the number of cultivation days increased (1-3 days), cytotoxicity tests of MG-63 osteoblast-like cells indicated no decrease in cell population. On Day 1, the cells were attached to the nitinol surface, and the cell shape changed from round to long-stripe-like and spindle-shaped, spread flatly on the surface. The number of cells was greater than that of the tissue culture plate control group. By Day 3, the cell population on the nitinol surface had increased substantially, indicating that the nitinol used in this study exhibited excellent biocompatibility.

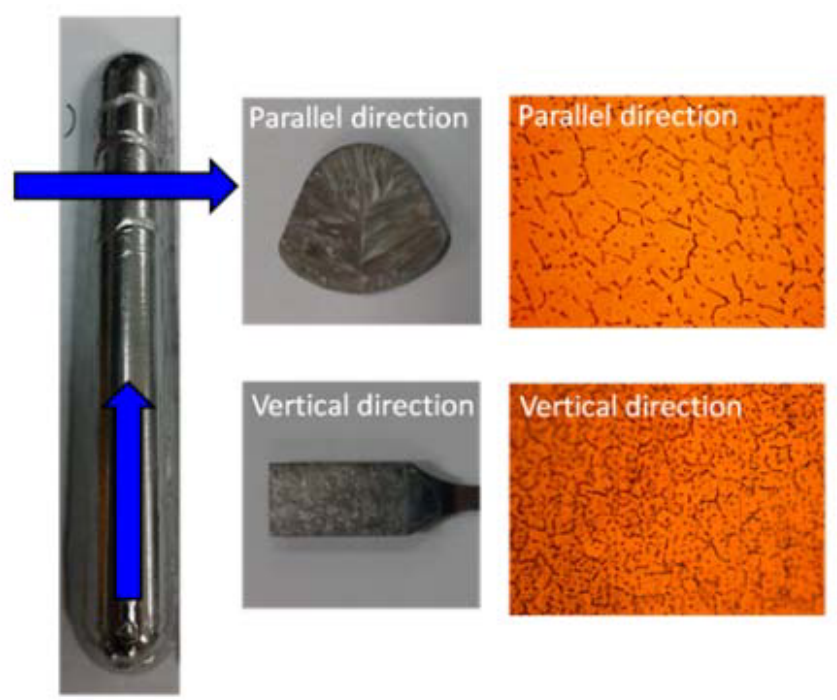

Fig.5 The metallograph of nitinol alloy

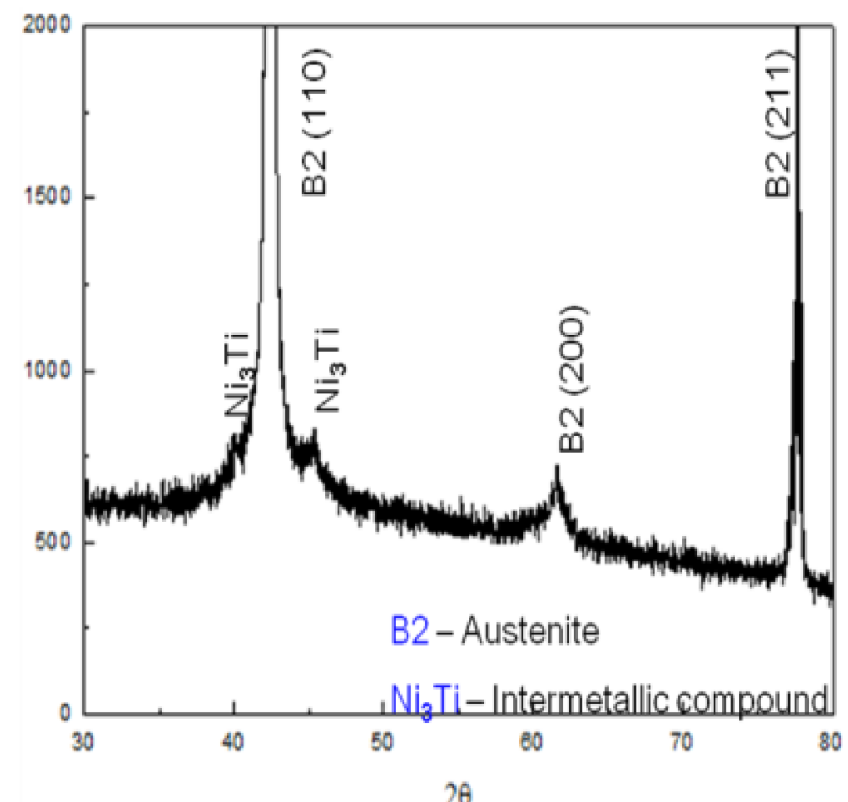

Fig.6 The XRD analysis of nitinol alloy 


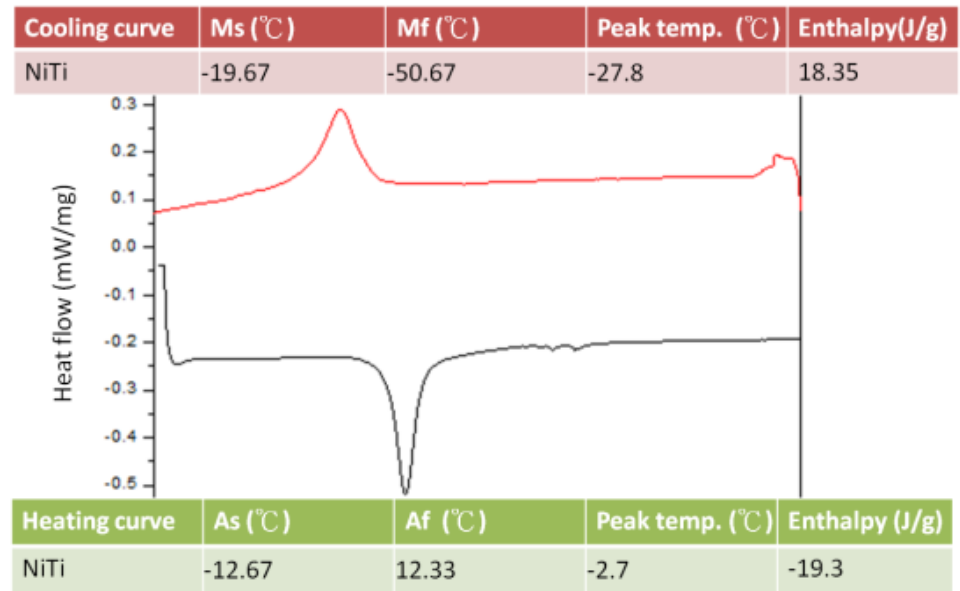

Fig.7 The DSC result of nitinol alloy

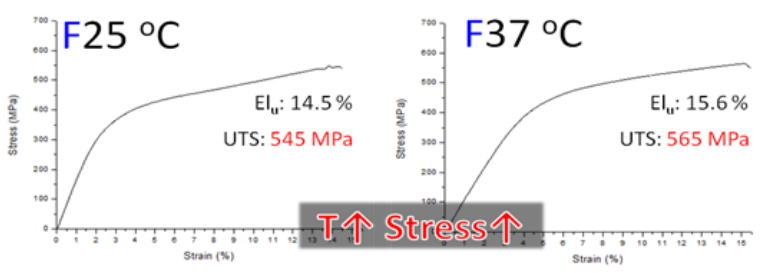

(a)
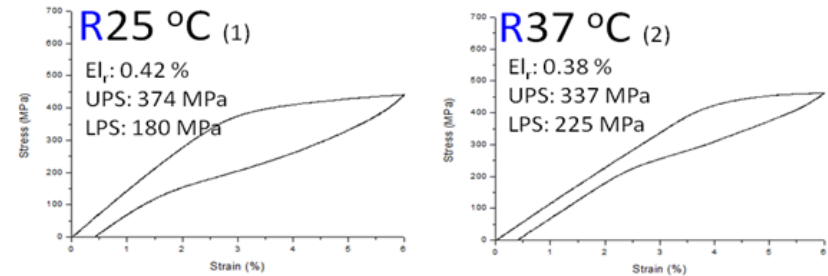

(b)

Fig.8 The tensile test of nitinol alloy

Cell cytotoxic test

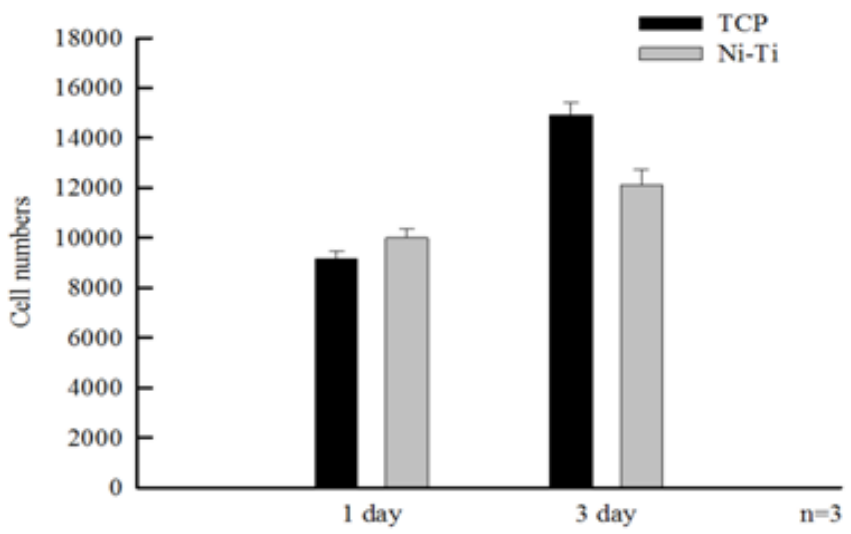

Fig.9 The Cytotoxicity test of nitinol alloy 


\section{MG-63 Osteoblast-like cell}

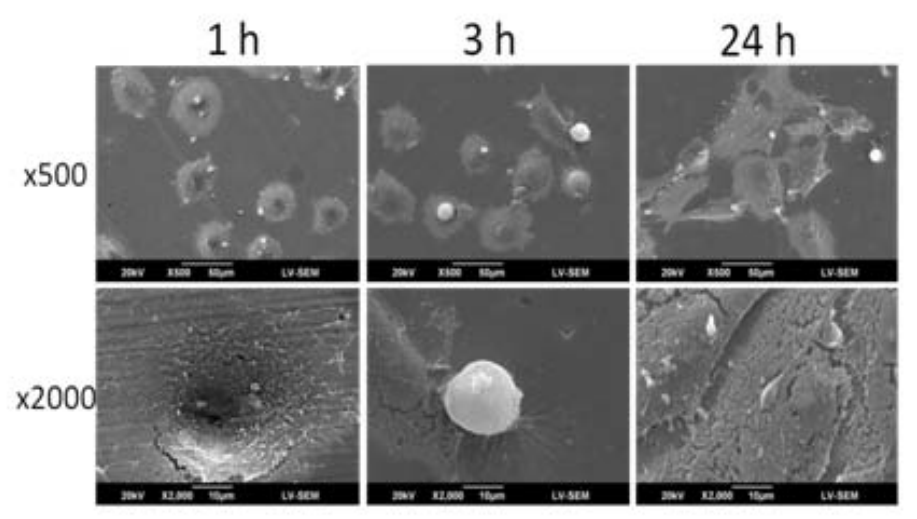

Fig.10 The observation of Cell culture of nitinol alloy

\section{Conclusion}

This study primarily focused on nitinol purification and melting technologies. Relevant property analyses were conducted to characterise the alloy ingot after VAR. The findings of this study are summarized as follows:

1. To analyse the alloy components, semiquantitative energy-dispersive X-ray spectroscopy was performed and a quantitative ICP analysis was conducted. After VAR, the gas and impurity content of the nitinol ingot satisfied the medical alloy specifications of ASTM F2063.

2. For the phase change analysis, a DSC thermal differential analysis was conducted. The results show that the nitinol ingot was in the austenite phase at room temperature.

3. The tensile strength test was conducted to analyse the mechanical properties of the alloy. The results show the elongation, tensile strength, and hyperelasticity of the nitinol ingot.
4. Regarding the biocompatibility analysis, cell culture tests were conducted. The results confirm that nitinol exhibits excellent biocompatibility.

\section{References}

1. A. Olander: Z. Krist.-Crystalline Materials Vol. 83 (1932), p. 145

2. H. C. Hong and C. M. Wayman: Acta Met. Vol. 22 (1974), p. 887.

3. C. M. Wayman: J. Metals Vol. 32 (1980), p. 129.

4. D. E. Hodgson, M. H. Wu and R. J. Biermann: Metals Handbook Vol. 2 10th Edition, Properties and Selection. ASM International.

5. J. Ryhanen, E. Niemi, W. Serlo and T. Salo: J Biomed Mater Res Vol. 35 (1997), p. 451.

6. D. J. Wever, A. G. Veldhuizen and M. Schakenard: Bomaterials Vol. 18 (1997), p. 115.

7. S. A. Shabalovskaya: Biomed Mater Eng Vol. 6 (1996), p. 267.

8. N. Figueira, T. M. Silva and J. C. S. Fernandes: Electrochemica Acta Vol. 54 (2009), p.921. 